Proyecciones Journal of Mathematics

Vol. 32, No 2, pp. 143-157, June 2013.

Universidad Católica del Norte

Antofagasta - Chile

\title{
The stability of fuzzy approximately Jordan mappings
}

\author{
N. Eghbali \\ University of Mohaghegh Ardabili, Iran \\ and \\ B. Farhadinia \\ Quchan Institute of Engineering and Technology, Iran \\ Received: December 2011. Accepted : March 2013
}

\begin{abstract}
In this paper we introduce the concept of fuzzy approximately Jordan mappings in fuzzy algebras, and study some of their basic properties. The main purpose of this paper is to study the stability of fuzzy approximately Jordan mappings in fuzzy algebras.
\end{abstract}

Subjclass : Primary 46S40; Secondary 39B52, 39B82, 26E50, 46S50.

Keywords : Fuzzy normed space; approximate Jordan map; stability. 


\section{Introduction}

The stability of functional equations is an interesting area of research for mathematicians, but it can be also of importance to persons who work outside of the realm of pure mathematics.

It seems that the stability problem of functional equations had been first raised by Ulam [11] which was answered in [4] and then generalized in [9].

Moreover the approximated mappings have been studied extensively in several papers. (See for instance [5], [6]).

Let $A$ and $B$ be Banach algebras and $\varphi: A \longrightarrow B$ a linear map. We say that $\varphi$ is an approximately Jordan map if there exists an $\epsilon>0$ such that for all $x \in A,\left\|\varphi\left(x^{2}\right)-\varphi(x)^{2}\right\| \leq \epsilon\|x\|^{2}$.

Fuzzy notion introduced firstly by Zadeh [12] that has been widely involved in different subjects of mathematics. Zadeh's definition of a fuzzy set characterized by a function from a nonempty set $X$ to $[0,1]$. Goguen in [3] generalized the notion of a fuzzy subset of $X$ to that of an $L$-fuzzy subset, namely a function from $X$ to a lattice $L$.

Later in 1984, Katsaras [7] defined a fuzzy norm on a linear space to construct a fuzzy vector topological structure on the space. Defining the class of approximate solutions of a given functional equation one can ask whether every mapping from this class can be somehow approximated by an exact solution of the considered equation in the fuzzy Banach algebra.

To answer this question, we use here the definition of fuzzy normed spaces given in [7] to exhibit some reasonable notions of fuzzy approximately additive and approximately Jordan functions in fuzzy normed algebras and we will prove that under some suitable conditions an approximately additive and approximately Jordan function $f$ from an algebra $X$ into a fuzzy Banach algebra $Y$ can be approximated in a fuzzy sense by an additive and Jordan mapping $T$ from $X$ to $Y$.

\section{Preliminaries}

In this section, we provide a collection of definitions and related results which are essential and used in the next discussions.

Definition 2.1. Let $X$ be a real linear space. A function $N: X \times \mathbf{R} \rightarrow$ $[0,1]$ is said to be a fuzzy norm on $X$ if for all $x, y \in X$ and all $t, s \in \mathbf{R}$,

(N1) $N(x, c)=0$ for $c \leq 0$;

(N2) $x=0$ if and only if $N(x, c)=1$ for all $c>0$; 
(N3) $N(c x, t)=N\left(x, \frac{t}{c \mid}\right)$ if $c \neq 0$;

(N4) $N(x+y, s+t) \geq \min \{N(x, s), N(y, t)\}$;

(N5) $N(x,$.$) is a non-decreasing function on \mathbf{R}$ and $\lim _{t \rightarrow \infty} N(x, t)=1$;

(N6) for $x \neq 0, N(x,$.$) is (upper semi) continuous on \mathbf{R}$.

The pair $(X, N)$ is called a fuzzy normed linear space.

Definition 2.2. Let $(X, N)$ be a fuzzy normed linear space and $\left\{x_{n}\right\}$ be a sequence in $X$. Then $\left\{x_{n}\right\}$ is said to be convergent if there exists $x \in X$ such that $\lim _{n \rightarrow \infty} N\left(x_{n}-x, t\right)=1$ for all $t>0$. In that case, $x$ is called the limit of the sequence $\left\{x_{n}\right\}$ and we denote it by $N-\lim _{n \rightarrow \infty} x_{n}=x$.

Definition 2.3. A sequence $\left\{x_{n}\right\}$ in $X$ is called Cauchy if, for each $\varepsilon>0$ and each $t>0$, there exists $n_{0}$ such that for all $n \geq n_{0}$ and all $p>0$, we have $N\left(x_{n+p}-x_{n}, t\right)>1-\varepsilon$.

It is known that every convergent sequence in a fuzzy normed space is Cauchy and if each Cauchy sequence is convergent, then the fuzzy norm is said to be complete and furthermore the fuzzy normed space is called a fuzzy Banach space.

Let $X$ be an algebra and $(X, N)$ be complete fuzzy normed space. The pair $(X, N)$ is said to be a fuzzy Banach algebra if for every $x, y \in X$ and $s, t \in \mathbf{R}$ we have: $N(x y, s t) \geq \min \{N(x, s), N(y, t)\}$.

Example 2.4. $\operatorname{Let}(X,\|\|$.$) be a Banach algebra. Define,$

$$
N(x, a)= \begin{cases}0, & a \leq\|x\| \\ 1, & a>\|x\| .\end{cases}
$$

Then $(X, N)$ is a fuzzy Banach algebra.

Theorem 2.5. Let $X$ be a linear space and $(Y, N)$ be a fuzzy Banach space. Let $\varphi: X \times X \rightarrow[0, \infty)$ be a control function such that

$$
\tilde{\varphi}(x, y)=\sum_{n=0}^{\infty} 2^{-n} \varphi\left(2^{n} x, 2^{n} y\right)<\infty,
$$

for all $x, y \in X$. Let $f: X \rightarrow Y$ be a uniformly approximately additive function with respect to $\varphi$ in the sense that

$$
\lim _{t \rightarrow \infty} N(f(x+y)-f(x)-f(y), t \varphi(x, y))=1
$$


uniformly on $X \times X$. Then $T(x)=N-\lim _{n \rightarrow \infty} \frac{f\left(2^{n} x\right)}{2^{n}}$ for all $x \in X$ exists and defines an additive mapping $T: X \rightarrow Y$ such that if for some $\delta>0$, $\alpha>0$

$$
N(f(x+y)-f(x)-f(y), \delta \varphi(x, y))>\alpha,
$$

for all $x, y \in X$; then

$$
N(T(x)-f(x), \delta / 2 \tilde{\varphi}(x, x))>\alpha,
$$

for every $x \in X$.

Proof. [8]

Corollary 2.6. Let $X$ be a linear space and $(Y, N)$ be a fuzzy Banach space. Let $\varphi: X \times X \rightarrow[0, \infty)$ be a control function such that

$$
\tilde{\varphi}(x, y)=\sum_{n=0}^{\infty} 2^{-n} \varphi\left(2^{n} x, 2^{n} y\right)<\infty,
$$

for all $x, y \in X$. Let $f: X \rightarrow Y$ be a uniformly approximately additive function with respect to $\varphi$. Then there is a unique additive mapping $T$ : $X \rightarrow Y$ such that

$$
\lim _{t \rightarrow \infty} N(f(x)-T(x), t \tilde{\varphi}(x, x))=1,
$$

uniformly on $X$.

Proof. [8]

Corollary 2.7. Let $X$ be a normed linear space and $(Y, N)$ a fuzzy Banach space. Let $\theta \geq 0$ and $0 \leq q<1$. Suppose that $f: X \rightarrow Y$ is a function such that

$$
\lim _{t \rightarrow \infty} N\left(f(x+y)-f(x)-f(y), t \theta\left(\|x\|^{q}+\|y\|^{q}\right)\right)=1
$$

uniformly on $X \times X$. Then there is a unique additive mapping $T: X \rightarrow$ $Y$ such that

$$
\lim _{t \rightarrow \infty} N\left(T(x)-f(x), \frac{2 \theta t|| x \|^{q}}{1-2^{q-1}}\right)=1
$$

uniformly on $X$. 


\section{Proof. [8]}

Remark 2.8. Using the sequence $\left\{2^{n} f\left(2^{-n} x\right)\right\}$, one can get dual version of Theorem 2.5 and Corollaries 2.6 and 2.7 when the control function satisfies

$$
\sum_{n=0}^{\infty} 2^{n} \varphi\left(2^{-n} x, 2^{-n} y\right)<\infty .
$$

In particular, the similar results hold for $\varphi(x, y)=\|x\|^{q}+\|y\|^{q}$, where $q>1$.

Theorem 2.9. Let $X$ be a linear space and let $\left(Z, N^{\prime}\right)$ be a fuzzy normed space. Let $\psi: X \times X \rightarrow Z$ be a function such that for some $0<\alpha<2$,

$$
N^{\prime}(\psi(2 x, 2 y), t) \geq N^{\prime}(\alpha \psi(x, y), t)
$$

for all $x, y \in X$ and $t>0$. Let $(Y, N)$ be a fuzzy Banach space and let $f: X \rightarrow Y$ be a mapping in the sense that

$$
N(f(x+y)-f(x)-f(y), t) \geq N^{\prime}(\psi(x, y), t)
$$

for each $t>0$ and $x, y \in X$. Then there exists unique additive mapping $T: X \rightarrow Y$ such that

$$
N(f(x)-T(x), t) \geq N^{\prime}\left(\frac{2 \psi(x, x)}{2-\alpha}, t\right),
$$

where $x \in X$ and $t>0$.

\section{Proof. [8]}

\section{Stability of fuzzy approximately Jordan mappings}

We start our work with definition of fuzzy approximately Jordan map and presenting some lemmas that are used in the proof of the main Theorem.

Definition 3.1. Let $X$ be a linear algebra, $(Y, N)$ a fuzzy Banach algebra and $\theta \geq 0$. We say that $f: X \rightarrow Y$ is a fuzzy approximately Jordan map if

$$
\lim _{t \rightarrow \infty} N\left(f\left(x^{2}\right)-f(x)^{2}, t \theta\|x\|^{2 q}\right)=1,
$$

uniformly on $X$.

Lemma 3.2. Let $(X, N)$ be a fuzzy normed linear space and $\left\{x_{n}\right\}$ a sequence in $X$. If $N-\lim _{n \rightarrow \infty} x_{n}=x$ for $x_{n}, x \neq 0$, then $N-\lim _{n \rightarrow \infty} c x_{n}=c x$ for every $c \in \mathbf{R}$. 
Proof. By definition we have $\lim _{t \rightarrow \infty} N\left(x_{n}-x, t\right)=1$ for all $t>0$. So $\lim _{t \rightarrow \infty} N\left(c x_{n}-c x, t\right)=\lim _{t \rightarrow \infty} N\left(c\left(x_{n}-x\right), t\right)=\lim _{t \rightarrow \infty} N\left(x_{n}-x, \frac{t}{c \mid}\right)=$ 1.

Theorem 3.3. Let $X$ be a normed linear algebra and $(Y, N)$ a fuzzy Banach algebra. Let $\theta \geq 0$ and $q \geq 0, q \neq 1$. Suppose that if $N-\lim _{n \rightarrow \infty} x_{n}=$ $x$ for $x_{n}, x=0$, then $N-\lim _{n \rightarrow \infty} x_{n}^{2}=x^{2}$ and also suppose that $f: X \rightarrow Y$ is a function such that $f(0)=0$,

$$
\lim _{t \rightarrow \infty} N\left(f(x+y)-f(x)-f(y), t \theta\left(\|x\|^{q}+\|y\|^{q}\right)\right)=1
$$

uniformly on $X \times X$ and

$$
\lim _{t \rightarrow \infty} N\left(f\left(x^{2}\right)-f(x)^{2}, t \theta\|x\|^{2 q}\right)=1
$$

uniformly on $X$. Then there is a unique Jordan additive mapping $T: X \rightarrow$ $Y$ such that

$$
\lim _{t \rightarrow \infty} N\left(T(x)-f(x), \frac{2 \theta t|| x \|^{q}}{\left|1-2^{q-1}\right|}\right)=1
$$

uniformly on $X$.

Proof. Theorem 2.5 and Corollaries 2.6 and 2.7 show that there exists a unique additive mapping $T$ such that for some $\delta>0, \alpha>0$

$$
N\left(T(x)-f(x), \frac{\theta \delta|| x \|^{q}}{\left|1-2^{q-1}\right|}\right) \geq \alpha,
$$

and

$$
\lim _{t \rightarrow \infty} N\left(T(x)-f(x), \frac{2 \theta t|| x \|^{q}}{\left|1-2^{q-1}\right|}\right)=1 .
$$

We first show that $T\left(a^{2}\right)=T(a)^{2}$ for all $a \in X$. If $a=0$ since $T(0)=0$ it is obvious. In the other case, there are two possibilities: $a^{2}=0$ or $a^{2} \neq 0$. In the first stage we suppose that $a^{2} \neq 0$. We will consider the case $a^{2}=0$ in the second stage. Pick $a \in X$ arbitrarily, and put $s=\frac{|1-q|}{1-q}$. Note that $s=1$ if $0 \leq q<1$ and $s=-1$ when $q>1$. Since $T$ is additive, it follows from Definition 3.1 that

$$
N\left(n^{-2 s} f\left(n^{2 s} a^{2}\right)-n^{-2 s} T\left(n^{2 s} a^{2}\right), \frac{n^{-2 s} \delta \theta\left\|n^{2 s} a^{2}\right\|^{q}}{\left|1-2^{q-1}\right|}\right) \geq \alpha .
$$

Now, since

$$
N\left(n^{-2 s} f\left(n^{2 s} a^{2}\right)-n^{-2 s} T\left(n^{2 s} a^{2}\right), \frac{n^{-2 s} \delta \theta\left\|n^{2 s} a^{2}\right\|^{q}}{\left|1-2^{q-1}\right|}\right)=
$$




$$
N\left(n^{-2 s} f\left(n^{2 s} a^{2}\right)-T\left(a^{2}\right), \frac{n^{-2 s} \delta \theta\left\|n^{2 s} a^{2}\right\|^{q}}{\left|1-2^{q-1}\right|}\right) .
$$

So,

$$
N\left(n^{-2 s} f\left(n^{2 s} a^{2}\right)-T\left(a^{2}\right), \frac{n^{-2 s} \delta \theta\left\|n^{2 s} a^{2}\right\|^{q}}{\left|1-2^{q-1}\right|}\right) \geq \alpha,
$$

for all $n \in \mathbf{N}$.

Similar to the above argument, one can show that

$$
N\left(n^{-s} f\left(n^{s} a\right)-T(a), \frac{n^{s(q-1)} \delta \theta|| a||^{q}}{\left|1-2^{q-1}\right|}\right) \geq \alpha
$$

for every $n \in \mathbf{N}$.

By virtue of 3.1 and 3.2, the latter inequalities state that:

$$
\lim _{t \rightarrow \infty} N\left(n^{-2 s} f\left(n^{2 s} a^{2}\right)-T\left(a^{2}\right), \frac{2 n^{2 s(q-1)} t \theta|| a^{2} \|^{q}}{\left|1-2^{q-1}\right|}\right)=1,
$$

and

$$
\lim _{t \rightarrow \infty} N\left(n^{-s} f\left(n^{s} a\right)-T(a), \frac{2 n^{s(q-1)} t \theta\|a\|^{q}}{\left|1-2^{q-1}\right|}\right)=1 .
$$

By assumption we get

$$
\lim _{t \rightarrow \infty} N\left(f\left(a^{2}\right)-f(a)^{2}, t \theta\|a\|^{2 q}\right)=1,
$$

so

$$
\lim _{t \rightarrow \infty} N\left(f\left(n^{2 s} a^{2}\right)-f\left(n^{s} a\right)^{2}, n^{2 s} t \theta\|a\|^{2 q}\right)=1,
$$

for all $n \in \mathbf{N}$. Hence

$$
\lim _{t \rightarrow \infty} N\left(n^{-2 s}\left(f\left(n^{2 s} a^{2}\right)-f\left(n^{s} a\right)^{2}\right), n^{2 s(q-1)} t \theta\|a\|^{2 q}\right)=1 .
$$

For all $t>0$ we have:

$N\left(T\left(a^{2}\right)-T(a)^{2}, t\right) \geq \min \left\{N\left(T\left(a^{2}\right)-n^{-2 s} f\left(n^{2 s} a^{2}\right), t / 3\right), N\left(n^{-2 s} f\left(n^{2 s} a^{2}\right)-\right.\right.$ $\left.\left.n^{-2 s} f\left(n^{s} a\right)^{2}, t / 3\right), N\left(n^{-2 s} f\left(n^{s} a\right)^{2}-T(a)^{2}, t / 3\right)\right\}$.

Given $\varepsilon>0$, we can find some $t_{1}>0$ such that

$$
N\left(T\left(a^{2}\right)-n^{-2 s} f\left(n^{2 s} a^{2}\right), t / 3\right) \geq 1-\varepsilon,
$$


for all $t / 3 \geq t_{1}$ and all $a \in X$.

Since $\lim _{n \rightarrow \infty} n^{2 s(q-1)} t \theta\|a\|^{2 q}=0$, there is some $n_{0}$ such that $n^{2 s(q-1)} t \theta\|a\|^{2 q}<t / 3$ for all $n \geq n_{0}$. On the other hand since $N$ is a nondecreasing function hence for each $n \geq n_{0}$,

$N\left(n^{-2 s}\left(f\left(n^{2 s} a^{2}\right)-f\left(n^{s} a\right)^{2}\right), t / 3\right)>N\left(n^{-2 s}\left(f\left(n^{2 s} a^{2}\right)-f\left(n^{s} a\right)^{2}\right)\right.$, $\left.n^{2 s(q-1)} t \theta\|a\|^{2 q}\right)$.

By the hypothesis, for given $\varepsilon>0$ we can find some $t_{2}>0$ such that

$$
N\left(n^{-2 s}\left(f\left(n^{2 s} a^{2}\right)-f\left(n^{s} a\right)^{2}\right), t / 3\right) \geq 1-\varepsilon,
$$

for all $t / 3 \geq t_{2}$ and all $a \in X$.

For given $\varepsilon>0$, we can find some $t_{3}>0$ such that

$$
N\left(n^{-2 s} f\left(n^{s} a\right)^{2}-T(a)^{2}, t / 3\right) \geq 1-\varepsilon,
$$

for all $t / 3 \geq t_{3}$ and all $a \in X$.

Let $t_{0}=\min \left\{t_{1}, t_{2}, t_{3}\right\}$. So $N\left(T\left(a^{2}\right)-T(a)^{2}, t\right) \geq 1-\varepsilon$ for all $t>0$, and by the item (N2) in the Definition 2.1 we have $T\left(a^{2}\right)=T(a)^{2}$.

Assume now that the later case holds, that is if $a \neq 0$ and $a^{2}=0$. It follows Corollary 2.7, that there exists an additive mapping $T: X \rightarrow Y$ such that

$$
\lim _{t \rightarrow \infty} N\left(f(x)-T(x), \frac{2 \theta t|| x \|^{q}}{\mid 1-2^{q-1}}\right)=1
$$

for all $x \in X$.

It suffices to show that $T\left(a^{2}\right)=T(a)^{2}$ for all $a \in X$. Pick $a \in X-\{0\}$ arbitrarily. In this case, we can not apply the pervious proof. In fact, if $a^{2}=0$ then $\left\|a^{2}\right\|^{q}=0$ and hence the relation 3.3 is meaningless by the item (N1) in the Definition 2.1. We will show that $T(a)^{2}=0$ whenever $a^{2}=0$. It follows from $\lim _{t \rightarrow \infty} N\left(f\left(a^{2}\right)-f(a)^{2}, t \theta\|a\|^{2 q}\right)=1$ and the hypothesis $f(0)=0$, that

$$
\lim _{t \rightarrow \infty} N\left(n^{-2} f(n a)^{2}, t \theta n^{-2}\|n a\|^{2 q}\right)=1 .
$$

Hence,

$$
\lim _{t \rightarrow \infty} N\left(n^{-2} f(n a)^{2}, t \theta n^{2(q-1)}\|a\|^{2 q}\right)=1 .
$$

Note also that

$$
\lim _{t \rightarrow \infty} N\left(f(x)-T(x), \frac{2 \theta t|| x \|^{q}}{\mid 1-2^{q-1}}\right)=1,
$$


and

$$
\lim _{t \rightarrow \infty} N\left(n^{-1} f(n a)-n^{-1} T(n a), \frac{2 \theta t|| n a \|^{q}}{\left|1-2^{q-1}\right|}\right)=1 .
$$

So we have $\lim _{t \rightarrow \infty} N\left(n^{-1} f(n a)-T(a), \frac{2 \theta t n^{q-1}|| a \|^{q}}{\left|1-2^{q-1}\right|}\right)=1$ for all $n \in \mathbf{N}$.

In the remaining of the proof we will take into consideration the following two cases:

Case 1. If $0 \leq q<1$, temporarily fix $t>0$. Given $\varepsilon>0$ we can find some $t_{1}>0$ such that $N\left(n^{-1} f(n a)-T(a), \frac{2 \theta t n^{q-1}|| a||^{q}}{\left|1-2^{q-1}\right|}\right) \geq 1-\varepsilon$ for all $t \geq t_{1}$. Since $\lim _{n \rightarrow \infty} \frac{2 t \theta n^{q-1}|| a||^{q}}{\left|1-2^{q-1}\right|}=0$, there is some $n_{0}$ such that $\frac{2 t \theta n^{q-1}|| a||^{q}}{\left|1-2^{q-1}\right|}<t$ for all $n \geq n_{0}$. Hence for all $n \geq n_{0}$

$$
N\left(n^{-1} f(n a)-T(a), t\right)>N\left(n^{-1} f(n a)-T(a), \frac{2 t \theta n^{q-1}|| a||^{q}}{\left|1-2^{q-1}\right|}\right) \geq 1-\varepsilon .
$$

We have $N-\lim _{n \rightarrow \infty} n^{-1} f(n a)=T(a)$. By Lemma ?? one gets

$$
N-\lim _{n \rightarrow \infty} n^{-2} f(n a)^{2}=T(a)^{2} .
$$

Given $\varepsilon>0$ we can find some $t_{2}>0$ such that $N\left(T(a)^{2}-n^{-2} f(n a)^{2}, t / 2\right) \geq$ $1-\varepsilon$ for all $t / 2 \geq t_{2}$. On the other hand for given $\varepsilon>0$ we can find some $t_{3}>0$ such that $N\left(n^{-2} f(n a)^{2}, t / 2\right) \geq 1-\varepsilon$ for all $t / 2 \geq t_{3}$. Hence taking $t_{0}=\min \left\{t_{1}, t_{2}, t_{3}\right\}$ we have

$N\left(T(a)^{2}-0, t\right) \geq \min \left\{N\left(T(a)^{2}-n^{-2} f(n a)^{2}, t / 2\right), N\left(n^{-2} f(n a)^{2}, t / 2\right)\right\} \geq$ $1-\varepsilon$.

This completes the proof for $0 \leq q<1$.

Case 2. If $q>1$, by assumption we have $\lim _{t \rightarrow \infty} N\left(f\left(a^{2}\right)-\right.$ $\left.f(a)^{2}, t \theta\|a\|^{2 q}\right)=1$ and since $f(0)=0$ it holds

$$
\lim _{t \rightarrow \infty} N\left(n^{2} f\left(n^{-1} a\right)^{2}, t \theta n^{2(1-q)}\|a\|^{2 q}\right)=1 .
$$

Note also that $\lim _{t \rightarrow \infty} N\left(n f\left(n^{-1} a\right)-n T\left(n^{-1} a\right), \frac{2 \theta t|| n^{-1} a||^{q}}{\left|1-2^{q-1}\right|}\right)=1$.

So $\lim _{t \rightarrow \infty} N\left(n f\left(n^{-1} a\right)-T(a), \frac{2 \theta t n^{1-q}|| a \|^{q}}{\left|1-2^{q-1}\right|}\right)=1$ for all $n \in \mathbf{N}$. Following an argument such as that of the proof of case 1 verifies case 2 .

Finally suppose that $T^{*}: X \rightarrow Y$ is another Jordan additive function such that $\lim _{t \rightarrow \infty} N\left(f(x)-T^{*}(x), \frac{2 \theta t|| x||^{q}}{\mid 1-2^{q-1}}\right)=1$ for all $x \in X$.

Then it holds

$$
\lim _{t \rightarrow \infty} N\left(n^{-s} f\left(n^{s} a\right)-T(a), \frac{2 n^{s(q-1)} t \theta|| a||^{q}}{\left|1-2^{q-1}\right|}\right)=1
$$


for $T=T^{*}$. We thus obtain

$N\left(T(a)-T^{*}(a), t\right) \geq \min \left\{N\left(T(a)-n^{-s} f\left(n^{s a}\right), t / 2\right), N\left(n^{-s} f\left(n^{s} a\right)-\right.\right.$ $\left.\left.T^{*}(a), t / 2\right)\right\}$.

Since $\lim _{n \rightarrow \infty} \frac{2 n^{s(q-1)} t \theta\|a\|^{q}}{\left|1-2^{q-1}\right|}=0$, there is some $n_{0}$ such that $\frac{2 n^{s(q-1)} t \theta \|\left. a\right|^{q}}{\left|1-2^{q-1}\right|}<t / 2$ for all $n \geq n_{0}$. Given $\varepsilon>0$, we can find some $t_{0}>0$ such that

$$
N\left(T(a)-n^{-s} f\left(n^{s a}\right), t / 2\right) \geq 1-\varepsilon
$$

and

$$
N\left(n^{-s} f\left(n^{s} a\right)-T^{*}(a), t / 2\right) \geq \varepsilon
$$

for all $t \geq t_{0}$. Hence $N\left(T(a)-T^{*}(a), t\right) \geq 1-\varepsilon$ for all $t>0$ and again by the item (N2) in the Definition 2.1, we have $T(a)=T^{*}(a)$.

Remark 3.4. Using the similar argument such as that of the proof of Theorem 3.3 one can get the similar results where $q<1$.

In the following example we will show that Theorem 3.3 does not necessarily hold for $q=1$.

Example 3.5. Let $X$ be a Banach algebra, $x_{0} \in X$ and $\alpha, \beta$ are real numbers such that $|\alpha| \geq 1-\|x\|^{2}$ and $\beta \leq\|x\|$, for every $x \in X$. Set

$$
f(x)=\alpha x+\beta x_{0}\|x\|, \quad(x \in X) .
$$

Moreover, for each fuzzy norm $N$ on $X$, we have

$$
\begin{gathered}
N(f(x+y)-f(x)-f(y), t(\|x\|+\|y\|)) \\
=N\left(\beta x_{0}(\|x+y\|-\|x\|-\|y\|), t(\|x\|+\|y\|)\right), \\
=N\left(\beta x_{0}, \frac{t(\|x\|+\|y\|)}{\|x+y\|-|x| \mid-\|y\|}\right) \geq N\left(\beta x_{0}, t\right) \quad(x, y \in X, \quad t \in \mathbf{R}) .
\end{gathered}
$$

Therefore by the item (N5) of the Definition 2.1, $\lim _{t \rightarrow \infty} N(f(x+y)-$ $f(x)-f(y), t(\|x\|+\|y\|))=1$, uniformly on $X \times X$.

Also

$$
\begin{gathered}
N\left(f\left(x^{2}\right)-f(x)^{2}, t\|x\|^{2}\right)= \\
N\left(\alpha x^{2}+\beta x_{0}\left\|x^{2}\right\|-\alpha^{2} x^{2}-\beta^{2} x_{0}^{2}\|x\|^{2}-2 \alpha \beta x x_{0}\|x\|,\right. \\
\left.t\|x\|^{2}\right) \geq \min \left\{N\left((1-\alpha) \alpha x^{2}, \frac{t\|x\|^{2}}{4}\right), N\left(\left\|x^{2}\right\| \beta x_{0}, \frac{t\|x\|^{2}}{4}\right)\right. \\
\left., N\left(-\beta^{2} x_{0}^{2}\|x\|^{2}, \frac{t\|x\|^{2}}{4}\right), N\left(-2 \alpha \beta x x_{0}\|x\|, \frac{t\|x\|^{2}}{4}\right)\right\}
\end{gathered}
$$


where $x \in X$ and $t \in \mathbf{R}$.

Taking into account the following inequalities

$$
\begin{gathered}
N\left((1-\alpha) \alpha x^{2}, \frac{t\|x\|^{2}}{4}\right)=N\left(\alpha x^{2}, \frac{t\|x\|^{2}}{4|1-\alpha|}\right) \geq N\left(\alpha x^{2}, t / 4\right), \\
N\left(\left\|x^{2}\right\| \beta x_{0}, \frac{t\|x\|^{2}}{4}\right)=N\left(\beta x_{0}, \frac{t\|x\|^{2}}{4\left\|x^{2}\right\|}\right) \geq N\left(\beta x_{0}, t / 4\right), \\
N\left(-\beta^{2} x_{0}^{2}\|x\|^{2}, \frac{t\|x\|^{2}}{4}\right)=N\left(\beta^{2} x_{0}^{2}, t\right) \geq N\left(\beta^{2} x_{0}^{2}, t / 4\right), \\
N\left(-2 \alpha \beta x_{0}\|x\|, \frac{t\|x\|^{2}}{4}\right)=N\left(2 \alpha x x_{0}, \frac{t\|x\|}{4|\beta|}\right) \geq N\left(2 \alpha x x_{0}, t / 4\right),
\end{gathered}
$$

it can be easily seen that $\lim _{t \rightarrow \infty} N\left(f\left(x^{2}\right)-f(x)^{2}, t\|x\|^{2}\right)=1$, uniformly on $X$ and therefore the conditions of Theorem 3.3 are fulfilled.

Now, we suppose that there exists a unique linear Jordan map $T$ satisfying the conditions of Theorem 3.3. By the equation

$$
\lim _{t \rightarrow \infty} N(f(x+y)-f(x)-f(y), t(\|x\|+\|y\|))=1 .
$$

For given $\varepsilon>0$, we can find some $t_{0}>0$ such that

$$
N(f(x+y)-f(x)-f(y), t(\|x\|+\|y\|)) \geq 1-\varepsilon,
$$

for all $x, y \in X$ and all $t \geq t_{0}$. By using the simple induction on $n$, we shall show that

$$
N\left(f\left(2^{n} x\right)-2^{n} f(x), \operatorname{tn} 2^{n}\|x\|\right) \geq 1-\varepsilon .
$$

Putting $y=x$ in 3.10, we get 3.11 for $n=1$. Let 3.11 holds for some positive integer $n$, then

$$
\begin{gathered}
N\left(f\left(2^{n+1} x\right)-2^{n+1} f(x), t(n+1) 2^{n+1}\|x\|\right) \geq \\
\min \left\{N\left(f\left(2^{n+1} x\right)-2 f\left(2^{n} x\right), t\left(\left\|2^{n} x\right\|+\left\|2^{n} x\right\|\right)\right), N\left(2 f\left(2^{n} x\right)-\right.\right. \\
\left.2^{n+1} f(x), 2 \operatorname{tn}\left(\left\|2^{n-1} x\right\|+\left\|2^{n-1} x\right\|\right)\right) \geq 1-\varepsilon .
\end{gathered}
$$

This completes the induction argument. We observe that

$$
\lim _{n \rightarrow \infty} N(T(x)-f(x), n t|| x \|) \geq 1-\varepsilon .
$$

Hence

$$
\lim _{n \rightarrow \infty} N(T(x)-f(x), n t\|x\|)=1 .
$$

One may regard $N(x, t)$ as the truth value of the statement 'the norm of $x$ is less than or equal to the real number t'. So 3.12 is a contradiction with the non-fuzzy sense. This means that there is no such a $T$. 


\section{Non-uniform type of stability of fuzzy approximately Jor- dan mappings}

We are in a position to give non-uniform type of Theorem 3.3.

Theorem 4.1. Let $X$ be a linear algebra, $\left(Z, N^{\prime}\right)$ a fuzzy Banach algebra and $\varphi: X \times X \rightarrow Z$ a function such that for some $0<\alpha<2$,

$$
N^{\prime}(\varphi(2 x, 2 y), t) \geq N^{\prime}(\varphi(x, y), t)
$$

for all $x, y \in X$ and $t>0$. Let $(Y, N)$ be a fuzzy Banach algebra and let $f: X \rightarrow Y$ be a function such that

$$
N(f(x+y)-f(x)-f(y), t) \geq N^{\prime}(\varphi(x, y), t),
$$

and

$$
N\left(f\left(x^{2}\right)-f(x)^{2}, s\right) \geq N^{\prime}(\varphi(x, x), s),
$$

for each $t, s>0$ and $x, y \in X$. Then there exists a unique additive Jordan mapping $T: X \rightarrow Y$ such that

$$
N(f(x)-T(x), t) \geq N^{\prime}\left(\frac{2 \varphi(x, x)}{2-\alpha}, t\right),
$$

where $x \in X$ and $t>0$.

Proof. Theorem 2.9 shows that there exists an additive function $T$ : $X \rightarrow Y$ such that

$$
N(f(x)-T(x), t) \geq N^{\prime}\left(\frac{2 \varphi(x, x)}{2-\alpha}, t\right),
$$

where $x \in X$ and $t>0$. Now we only need to show that $T$ is a Jordan map. If $a=0$, since $T(0)=0$ it is obvious. In the other case,

$$
N\left(n^{-2} f\left(n^{2} a^{2}\right)-n^{-2} T\left(n^{2} a^{2}\right), n^{-2} t\right) \geq N^{\prime}\left(\frac{2 \varphi\left(n^{2} a^{2}, n^{2} a^{2}\right)}{2-\alpha}, t\right),
$$

for all $a \in X, t>0$ and $n \in \mathbf{N}$. by the additivity of $T$ it is easy to see that

$$
N\left(n^{-2} f\left(n^{2} a^{2}\right)-T\left(a^{2}\right), t\right) \geq N^{\prime}\left(\frac{2 \varphi\left(n^{2} a^{2}, n^{2} a^{2}\right)}{2-\alpha}, n^{2} t\right),
$$

for all $a \in X, t>0$ and $n \in \mathbf{N}$. Letting $n$ tend to infinity in 4.1 and using the items (N2) and (N5) of the Definition 2.1, we see that

$$
T\left(a^{2}\right)=N-\lim _{n \rightarrow \infty} n^{-2} f\left(n^{2} a^{2}\right) .
$$

Also with a similar argument represented above shows that: 


$$
N\left(n^{-1} f(n a)-T(a), t\right) \geq N^{\prime}\left(\frac{2 \varphi(n a, n a)}{2-\alpha}, n t\right),
$$

for all $a \in X, t>0$ and $n \in \mathbf{N}$. Hence we have

$$
T(a)=N-\lim _{n \rightarrow \infty} n^{-1} f(n a) .
$$

On the other hand

$$
N\left(f\left(n^{2} a^{2}\right)-f(n a)^{2}, s\right) \geq N^{\prime}(\varphi(n a, n a), s)
$$

for all $a \in X, s>0$ and $n \in \mathbf{N}$. We observe that

$$
N\left(n^{-2} f\left(n^{2} a^{2}\right)-n^{-2} f(n a)^{2}, s\right) \geq N^{\prime}\left(\varphi(n a, n a), n^{2} s\right),
$$

for all $a \in X, s>0$ and $n \in \mathbf{N}$. So again by taking $n$ tend to infinity we have

$$
N-\lim _{n \rightarrow \infty} n^{-2} f\left(n^{2} a^{2}\right)=N-\lim _{n \rightarrow \infty} n^{-2} f(n a)^{2} .
$$

Applying 4.2, 4.3 and 4.4 we have

$$
\begin{gathered}
T\left(a^{2}\right)=N-\lim _{n \rightarrow \infty} n^{-2} f\left(n^{2} a^{2}\right)=N-\lim _{n \rightarrow \infty} n^{-2} f(n a)^{2}= \\
\left(N-\lim _{n \rightarrow \infty} n^{-1} f(n a)\right)^{2}=T(a)^{2} .
\end{gathered}
$$

To prove the uniqueness property of $T$, assume that $T^{*}$ is another additive Jordan mapping satisfying $N(f(x)-T(x), t) \geq N^{\prime}\left(\frac{2 \varphi(x, x)}{2-\alpha}, t\right)$. Since both $T$ and $T^{*}$ are additive we deduce that

$$
\begin{gathered}
N\left(T(a)-T^{*}(a), t\right) \geq \min \left\{N\left(T(a)-n^{-1} f(n a), t / 2\right), N\left(n^{-1} f(n a)-\right.\right. \\
\left.\left.T^{*}(a), t / 2\right)\right\} \geq N^{\prime}\left(\frac{2 \varphi(n a, n a)}{2-\alpha}, n t / 2\right)
\end{gathered}
$$

for all $a \in X$ and all $t>0$. Letting $n$ tend to infinity we find that $T(a)=T^{*}(a)$ for all $a \in X$.

\section{References}

[1] Z. Gajda, On stability of additive mappings, Intermat. J. Math. Sci., 14, pp. 431-434, (1991). 
[2] P. Gavruta, A generalization of the Hyers-Ulam-Rassias stability of approximately additive mappings, J. Math. Anal. Appl., 184, pp. 431436, (1994).

[3] J. A. Goguen, L-fuzzy sets, J. Math. Anal. Appl., 18, pp. 145-174, (1967).

[4] D. H. Hyers, On the stability of the linear functional equation, Proc. Natl. Acad. Sci., U.S.A. 27, pp. 222-224, (1941).

[5] D. H. Hyers, G.Isac and Th. M. Rassias, Stability of functional equations in several variables, Birkhäuser, Basel, (1998).

[6] D. H. Hyers and Th. M. Rassias, Approximate homomorphisms, Aequationes Math. 44 (2-3), pp. 125-153, (1992).

[7] A. K. Katsaras, Fuzzy topological vector spaces II, Fuzzy Sets and Systems, 12, pp. 143-154, (1984).

[8] A. K. Mirmostafaee and M. S. Moslehian, Fuzzy versions of HyersUlam-Rassias theorem, Fuzzy Sets and Systems, 159 (6), pp. 720-729, (2008).

[9] Th. M. Rassias, On the stability of the linear mapping in Banach spaces, Proc. Amer. Math. Soc., 72, pp. 297-300, (1978).

[10] Th. M. Rassias and P. Šemrl, On the behavior of mappings which do not satisfy Hyers-Ulam stability, Proc. Amer. Math. Soc., 173, pp. 325-338, (1993).

[11] S. M. Ulam, Problems in modern mathematics, Chap. VI, Science eds., wiley, New York, (1960).

[12] L. A. Zadeh, Fuzzy sets, Inform. and Control, 8, pp. 338-353, (1965).

\author{
N. Eghbali \\ Department of Mathematics, \\ Facualty of Mathematical Sciences, \\ University of Mohaghegh Ardabili, \\ 56199-11367, Ardabil, \\ Iran \\ e-mail : eghbali@uma.ac.ir
}


and

B. Farhadinia

Department of Mathematics,

Quchan Institute of Engineering and Technology,

Quchan,

Iran

e-mail : bfarhadinia@yahoo.com.au 